\title{
Launching the chaotic realm of iso-fractals: a short remark
}

\author{
Nathan O. Schmidt*, Reza Katebi ${ }^{\dagger}$ and Christian Corda** \\ *Department of Mathematics, Boise State University, 1910 University Drive, Boise, ID 83725, USA \\ ${ }^{\dagger}$ Department of Physics, California State University in Fullerton, 800 North State College Boulevard, Fullerton, \\ CA 92831, USA \\ **Institute for Theoretical Physics and Advanced Mathematics Einstein-Galilei (IFM), Via Santa Gonda 14, \\ 59100 Prato, Italy
}

\begin{abstract}
In this brief note, we introduce the new, emerging sub-discipline of iso-fractals by highlighting and discussing the preliminary results of recent works. First, we note the abundance of fractal, chaotic, non-linear, and self-similar structures in nature while emphasizing the importance of studying such systems because fractal geometry is the language of chaos. Second, we outline the iso-fractal generalization of the Mandelbrot set to exemplify the newly generated Mandelbrot iso-sets. Third, we present the cutting-edge notion of dynamic iso-spaces and explain how a mathematical space can be iso-topically lifted with iso-unit functions that (continuously or discretely) change; in the discrete case examples, we mention that iteratively generated sequences like Fibonacci's numbers and (the complex moduli of) Mandelbrot's numbers can supply a deterministic chain of iso-units to construct an ordered series of (magnified and/or de-magnified) iso-spaces that are locally iso-morphic. Fourth, we consider the initiation of iso-fractals with Inopin's holographic ring (IHR) topology and fractional statistics for 2D and 3D iso-spaces. In total, the reviewed iso-fractal results are a significant improvement over traditional fractals because the application of Santilli's iso-mathematics arms us an extra degree of freedom for attacking problems in chaos. Finally, we conclude by proposing some questions and ideas for future research work.
\end{abstract}

Keywords: Fractals, Chaos theory, Mandelbrot set, Iso-mathematics

PACS: 05.45.Df, 05.45.Gg

Chaos theory is the behavioral study of dynamical systems that are highly sensitive to initial conditions [1,2]. In a chaotic dynamical system, minuscule differences in initial conditions inevitably yield expansively divergent outcomes, which generally renders the long-term predictability of such systems impossible [1, 2]. The abundance of chaos in nature is becoming more evident $[2,3,4,5,6]$ and the patterns exemplified in such complex, non-linear systems are widely examined in disciplines like mathematics $[5,7,8]$, physics $[9,10,11]$, gravitation and cosmology [12, 13], astrophysics [14, 15], chemistry [16, 17, 18], biology [19, 20, 21, 22, 23, 24, 25, 26], neuro-science [27, 28, 29], medicine [30, 31], computation [32, 33, 34], cryptology [35, 36, 37], economics [38, 39], and warfare [40, 41, 42]. In general, chaos theory has substantially improved our scientific comprehension of phenomena ranging from turbulence to structural dynamics [1, 2, 40, 43] which applies to, for example, aquatic ecosystems [44], weather [45], black holes [9], the cosmic microwave background [13], galaxy distributions [14, 15], population biology [19], viruses and pathogens [20], cancers and genetics [21, 46], military strategy [40, 41, 42], volcanoes [47], earthquakes [48, 49], and the global stock market [38]. Hence, it is crucial to scrutinize such phenomenon from diverse perspectives and attack chaotic problems from multiple directions.

Now a paramount and fundamental fact to know is that fractals are the language of chaos theory [50, 51, 52]. Thus, if we wish to gain applicable knowledge and advance our scientific awareness of chaos and nature, then it is surely logical to study, develop, and upgrade fractal geometry via the scientific method in order to equip ourselves with a suitable language for further characterizing self-similar patterns and power laws in the universe. Moreover, one must also note that incredibly complex fractal, chaotic, non-linear, and self-similar structures can be generated on a computer with relatively simple iterated functions $[5,6]$; in our opinion, this cornerstone relation between simplicity and complexity delivers fractal geometry as an appealing candidate for the study and application of chaotic dynamical systems and power law functions. Hence, in this extended abstract, we start by highlighting some key aspects of recent, preliminary work that sparks the new, emerging sub-discipline of iso-fractals [53, 54, 55], where Santilli's iso-mathematics $[56,57,58,59,60]$ is introduced to the domain of fractals and chaos.

In Ref. [53], the authors computationally generate the planet's first iso-fractal, namely the Mandelbrot iso-set, which is an iso-mathematical $[56,57,58,59,60]$ generalization of the famous Mandelbrot set [5, 6]. For this, Mandelbrot's 

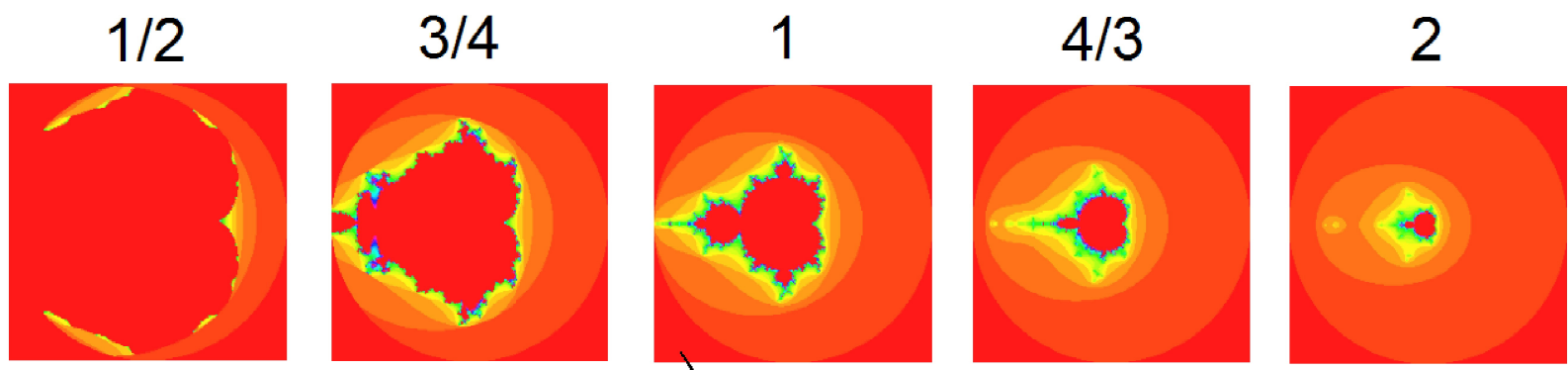

\section{Control}

FIGURE 1. A depiction of the experimental results of Ref. [53], where the five distinct iso-unit impact variations for $\hat{r}=$ $\frac{1}{2}, \frac{3}{4}, 1, \frac{4}{3}, 2$ are displayed. In the control case of $\hat{r}=1$ the traditional Mandelbrot set is restored (the middle graphic), whereas for $\hat{r}=\frac{1}{2}, \frac{3}{4}, \frac{4}{3}, 2$ the locally iso-morphic Mandelbrot iso-sets are generated and the scale-deformation and boundary-deformation effects are exemplified [53]. In the top row the values for $\hat{r}$ are listed and in the bottom row the Mandelbrot iso-sets [53] are displayed.

well known complex quadratic polynomial [5, 6]

$$
z_{n+1}=z_{n}^{2}+c
$$

is first iso-topically lifted [56, 57, 58, 59, 60] in the procedure of Ref. [53] to define Mandelbrot's new iso-complex quadratic polynomial as [53]

$$
\hat{z}_{n+1}=\hat{z}_{n}^{2}+\hat{c}
$$

from eq. (7) of Ref. [53], where the $z_{n} \times z_{n}$ multiplication of eq. (1) is replaced with the $\hat{z}_{n} \hat{\times} \hat{z}_{n}$ iso-multiplication of eq. (2) via the procedure of Ref. [53], such that the positive-definite iso-unit $\hat{r}>0$ (with its corresponding inverse $\hat{k}=\frac{1}{\hat{r}}$ ) is selected and applied in accordance to Santilli's iso-topic liftings [56, 57, 58, 59, 60]. Next, the algorithm for eq. (2) is programmed into a computer and the experiment is conducted for the five distinct iso-units $\hat{r}=\frac{1}{2}, \frac{3}{4}, 1, \frac{4}{3}, 2$ in eq. (8) of Ref. [53] to computationally generate the five resulting Mandelbrot iso-set instances of Figure 1 in order to give a preliminary assessment of the resulting iso-topic lifting impact on eq. (2) when $\hat{r}$ is varied [53]. As we observe in the preliminary iso-unit impact variation results of Figure 1, the iso-topic liftings [56, 57, 58, 59, 60] impose (at least) two general topological effects [53]:

1. scale-deformation, where the fractal is magnified ("zoom-in”) or de-magnified ("zoom-out") [53]; and

2. boundary-deformation, where the relative position of the fractal boundaries and sequence divergence rates are restructured [53].

Hence, the initial scale-deformation and boundary-deformation results (as displayed in Figure 1) identified in the experiments [53] prove that the Mandelbrot set "control case" of eq. (1) can be generalized with iso-mathematics $[56,57,58,59,60]$ for $0<\hat{r} \neq 1$ to create the new locally iso-morphic Mandelbrot iso-sets of eq. (2), where in the control case of $\hat{r}=1$ the traditional Mandelbrot set is restored. Therefore, the said Mandelbrot iso-sets [53] are significantly more general than typical Mandelbrot sets because the iso-unit $[56,57,58,59,60]$ serves as an extra degree of freedom to work with [53] - this iso-mathematical generalization is an improvement that broadens and strengthens the chaotic analysis by initiating an enhanced classification and demystification of such complex systems [53].

Now Santilli showed that the iso-units can be a number of different mathematical constructions, including positivedefinite functions that vary [56, 57, 58, 59, 60]. Hence, in Ref. [54] the author focused on this notion and deployed Santilli's iso-unit functions $[56,57,58,59,60]$ to construct and investigate dynamic iso-spaces, which are iso-spaces $[56,57,58,59,60]$ built with dynamic iso-topic liftings that are characterized by iso-unit functions that vary over "time"; so if $\hat{r}$ is the iso-unit, $n$ is the time (or $n$th iteration index), and $f(n)$ is the iso-unit function, then we have [54]

$$
\hat{r}=f(n)>0 \text { and } \hat{\kappa}=\frac{1}{\hat{r}}
$$

in generic form. In this preliminary exploration [54], both the continuous and discrete cases were considered but the primary focus was on discrete dynamic iso-topic liftings with a direct application to fractal and self-similar structures. 
Hence, the iso-unit function of eq. (3) can be continuous or discrete, depending on the application context [54]. For this, two simple examples were presented and defined [54]:

1. a Fibonacci dynamic iso-space is a discrete dynamic iso-space that is iso-topically lifted $[56,57,58,59,60]$ with the positive-definite, ever-increasing Fibonacci sequence iso-unit values [54]

$$
\hat{r} \in\{f(1)=1, f(2)=1, f(3)=2, f(4)=3, f(5)=5, f(6)=8, f(7)=13, \ldots\}
$$

and

2. a Mandelbrot dynamic iso-space is a discrete dynamic iso-space that is iso-topically lifted with the positivedefinite complex number moduli of Mandelbrot set points (or "Mandelbrot numbers") [54]

$$
\hat{r} \in\left\{f(n)=\left|z_{n}\right|, f(n+1)=\left|z_{n+1}\right|, f(n+2)=\left|z_{n+2}\right|, f(n+3)=\left|z_{n+3}\right|, \ldots\right\},
$$

which are sequentially generated by iterating Mandelbrot's complex quadratic polynomial [5, 6] of eq. (1), such that $\left|z_{n}\right|$ is the modulus of the $n$th complex number $z_{n}$.

The central idea here is that the iso-units returned by the iso-unit functions of eqs. (4-5) can be utilized to iso-topically lift $[56,57,58,59,60]$ a space $X$ via the sequential iterations

$$
X \rightarrow X_{1} \rightarrow X_{2} \rightarrow X_{3} \rightarrow X_{4} \rightarrow \ldots \rightarrow X_{n} \rightarrow \ldots
$$

which can also be reversed when the iso-unit inverses are iso-topically applied [54] because all such instances are locally iso-morphic. If the Fibonacci case of eq. (4) is applied to $X$, then the ever-increasing Fibonacci iso-units will always magnify it, whereas if the Mandelbrot case of eq. (5) is applied to $X$, then sometimes it will be magnified and other times it will be de-magnified. Furthermore, it is interesting to inquire the following: can the Fibonacci case of eq. (4) —or some variation of it—be deployed to model some ever-expanding, ever-accelerating, spiral-like aspects of the universe?

Finally, we highlight the preliminary iso-fractal work of Ref. [55] (and suggest some possible ideas for future exploration), where Santilli's iso-mathematics $[56,57,58,59,60]$ is initiated to iso-fractals in $2 \mathrm{D}$ and $3 \mathrm{D}$ versions of the IHR topology [61]. In short, the 2D IHR topology [55, 61] is a 2D/complex space $X$ (i.e. Riemann surface or similar compatible structure) equipped with 2D Cartesian-polar generalized coordinate-vectors, where from eq. (16) in Ref. [55] the topological 1-sphere IHR

$$
T^{1}=\{\vec{x} \in X:|\vec{x}|=r\}
$$

of amplitude-radius $r$ (and corresponding curvature $\kappa=\frac{1}{r}$ ) is centered on the origin and iso-metrically embedded in $X$, such that $T^{1} \subset X$ is a scaled Riemannian circle that simultaneously delineates the disjoint "micro-space zone" $X_{-} \subset X$ and the "macro-space zone" $X_{+} \subset X[55,61]$ in accordance to the 2D holographic version of M.C. Escher's famous reflecting sphere and duality [62]. Since $X$ is a complex space it was discussed [55] that 2D fractals such as the Mandelbrot set [5,6] could be populated in $X$. Moreover, given the said IHR topology with $X_{-} \cup T^{1} \cup X_{+}=X$ $[55,61]$, it would be interesting to see if a "micro" Mandelbrot set $[5,6]$ could be computationally generated in $X_{-}$and a corresponding "macro" Mandelbrot set [5, 6] could be generated in $X_{+}$, with respect to an Escher reflection [55, 61] over $T^{1}$. Furthermore, we know that such fractals $[5,6]$ can be iso-topically lifted [56, 57, 58, 59, 60] to iso-fractals $[53,54,55]$ and that self-similar, chaotic structures can be expressed in terms of fractional quantities, therefore we propose the following question: if the IHR topology of $X[55,61]$ is equipped with fractional statistics like in Ref. [61], can such fractional quantities be connected to iso-fractals and power laws with iso-topic liftings $[56,57,58,59,60]$ ? Is there a direct physical application? Moreover, for a generalization from $2 \mathrm{D}$ to $3 \mathrm{D}$, if we consider a topological 2-sphere IHR $T^{2}$ from eq. (33) in Ref. [55], such that $T^{1} \subset T^{2}$ becomes the great circle of $T^{2} \subset Y$ in the 3D space $Y$, then we present an additional question: can the 2D fractional statistics and 2D iso-fractals in $X$ be generalized or "projected" to $Y$ for a 3D case? In our opinion, these should be interesting questions to follow-up on in near future research because fractional statistics, fractals, and chaos are abundant in the universe.

\section{REFERENCES}

1. S. H. Kellert, In the wake of chaos: Unpredictable order in dynamical systems, University of Chicago Press, 1993.

2. E. N. Lorenz, The essence of chaos, Routledge, 1995. 
3. G. W. Flake, The computational beauty of nature: Computer explorations of fractals, chaos, complex systems and adaption, The MIT Press, 1998.

4. J. Briggs, Fractals: The patterns of chaos: A new aesthetic of art, science, and nature, Touchstone Books, 1992.

5. B. B. Mandelbrot, The fractal geometry of nature, Times Books, 1982.

6. B. B. Mandelbrot, Fractals and chaos: the Mandelbrot set and beyond, vol. 3, Springer, 2004.

7. I. Stewart, Does God play dice?: The new mathematics of chaos, Penguin UK, 1997.

8. H. Nagashima, and Y. Baba, Introduction to chaos: physics and mathematics of chaotic phenomena, CRC Press, 2002.

9. L. Bombelli, and E. Calzetta, Classical and Quantum Gravity 9, 2573-2599 (1992).

10. M. El Naschie, Chaos, Solitons \& Fractals 29, 23-35 (2006).

11. S. H. Strogatz, Nonlinear dynamics and chaos: with applications to physics, biology and chemistry, Perseus publishing, 2001.

12. X. Wu, and T. Huang, Physics Letters A 313, 77-81 (2003).

13. L. B. Crowell, and C. Corda, Galaxies 2, 160-188 (2014).

14. J. Gaite, and S. C. Manrubia, Monthly Notices of the Royal Astronomical Society 335, 977-983 (2002).

15. N. Sánchez, and E. J. Alfaro, The Astrophysical Journal Supplement Series 178, 1-19 (2008).

16. B. Peng, V. Petrov, and K. Showalter, The Journal of Physical Chemistry 95, 4957-4959 (1991).

17. R. J. Field, and L. Györgyi, Chaos in chemistry and biochemistry, World Scientific, 1993.

18. P. W. Hoskin, Geochimica et Cosmochimica Acta 64, 1905-1923 (2000).

19. P. Philippe, Human Biology 65, 525-46 (1993).

20. L. Bos, and et al., Plant viruses, unique and intriguing pathogens: a textbook of plant virology., Backhuys Publishers, 1999.

21. P. Duesberg, Scientific American Magazine 296, 52-59 (2007).

22. G. B. West, J. H. Brown, and B. J. Enquist, Science 276, 122-126 (1997).

23. G. B. West, J. H. Brown, and B. J. Enquist, Science 284, 1677-1679 (1999).

24. R. Wu, C. X. Ma, R. C. Littell, and G. Casella, Journal of theoretical biology 219, 121-135 (2002).

25. G. B. West, and J. H. Brown, Journal of Experimental Biology 208, 1575-1592 (2005).

26. L. Demetrius, Journal of theoretical biology 243, 455-467 (2006).

27. C. A. Skarda, and W. J. Freeman, Concepts in Neuroscience 1, 275-285 (1990).

28. S. J. Schiff, K. Jerger, D. H. Duong, T. Chang, M. L. Spano, W. L. Ditto, et al., Nature 370, 615-620 (1994).

29. H. Haken, Principles of brain functioning: A synergetic approach to brain activity, behavior and cognition, Springer Publishing Company, Incorporated, 2012.

30. D. S. Coffey, Nature medicine 4, 882-885 (1998).

31. B. J. West, Fractal physiology and chaos in medicine, vol. 16, World Scientific, 2013.

32. J. P. Crutchfield, and K. Young, "Computation at the onset of chaos," in The Santa Fe Institute, Westview, Citeseer, 1988.

33. R. L. Devaney, Chaos and fractals: The mathematics behind the computer graphics, American Mathematical Soc., 1989.

34. N. Bertschinger, and T. Natschläger, Neural computation 16, 1413-1436 (2004).

35. L. Kocarev, Circuits and Systems Magazine, IEEE 1, 6-21 (2001).

36. G. Jakimoski, L. Kocarev, et al., IEEE Transactions on Circuits and Systems I: Fundamental Theory and Applications $\mathbf{4 8 ,}$ 163-169 (2001).

37. M. A. Alia, and A. Samsudin, American Journal of Applied Sciences 4, 850-858 (2007).

38. B. LeBaron, and B. LeBaron, Philosophical Transactions of the Royal Society of London. Series A: Physical and Engineering Sciences 348, 397-404 (1994).

39. J. B. Rosser, From Catastrophe to Chaos: A General Theory of Economic Discontinuities: Mathematics, Microeconomics and Finance, vol. 1, Springer, 2000.

40. D. Nicholls, T. Tagarev, and P. Axup, Airpower Journal pp. 48-57 (1994).

41. G. E. James, Chaos Theory: The Essentials for Military Applications, 10, Naval War College, Center for Naval Warfare Studies, 1996.

42. A. Bousquet, The scientific way of warfare: Order and chaos on the battlefields of modernity, vol. 1, Cinco Puntos Press, 2009.

43. J. Gleick (2008).

44. J. Huisman, and F. J. Weissing, Nature 402, 407-410 (1999).

45. A. A. Tsonis, and J. B. Elsner, Bulletin of the American Meteorological Society 70, 14-23 (1989).

46. G. A. Calin, C. Vasilescu, M. Negrini, and G. Barbanti-Brodano, Medical hypotheses 60, 258-262 (2003).

47. S. L. Harris, Agents of chaos: earthquakes, volcanoes, and other natural disasters, Mountain Press Publishing Company, 1990.

48. H. J. Xu, and L. Knopoff, Physical Review E 50, 3577-3581 (1994).

49. C. H. Scholz, Nature 348, 197-198 (1990).

50. D. Larsen-Freeman, Applied linguistics 18, 141-165 (1997).

51. H. O. Peitgen, H. Jürgens, and D. Saupe, Chaos and fractals: new frontiers of science, Springer, 2004.

52. M. R. Schroeder, Fractals, chaos, power laws: Minutes from an infinite paradise, Courier Dover Publications, 2012.

53. R. Katebi, and N. O. Schmidt, Hadronic Journal 36, 211-225 (2013).

54. N. O. Schmidt, Hadronic Journal 36, 167-176 (2013).

55. N. O. Schmidt, and R. Katebi, Accepted by the Hadronic Journal (viXra:1308.0051) (2013).

56. R. M. Santilli, Algebras, Groups and Geometries 10, 273-322 (1993).

57. R. M. Santilli, Supplemento 42, 7-16 (1996). 
58. C. X. Jiang, International Academic Presss, America-Europe-Asia (2002).

59. C. Corda, "Introduction to Santilli iso-numbers," in AIP Conference Proceedings-American Institute of Physics, 2012 , vol. 1479, p. 1013.

60. C. Corda, "Introduction to Santilli iso-mathematics," in AIP Conference Proceedings-American Institute of Physics, 2013, vol. 1558, p. 685.

61. A. E. Inopin, and N. O. Schmidt, Hadronic Journal 35, 469-507 (2012).

62. M. C. Escher, B. Ernst, and J. E. Brigham, The magic mirror of MC Escher, Random House, 2007. 